\title{
Ansia por la comida y alimentación emocional: variables asociadas
}

Sebastián Díaz Pérez - Universidad de Granada

Ana Mํㅡㄹ Beltrán-Morillas - Universidad de Granada
0000-0003-1273-2930

D 0000-0001-8950-1853

Recepción: 22.09.2021 | Aceptado: 16.10.2021

Correspondencia a través de ORCID: Sebastián Díaz Pérez

iD 0000-0003-1273-2930

Citar: Díaz Pérez, S y Beltrán morillas, AM (2021). Ansia por la comida y alimentación emocional: variables asociadas. REIDOCREA, 10(37), 1-10.

Área o categoría del conocimiento: Multidisciplinar

Resumen: El ansia por la comida y la alimentación emocional se consideran dos problemáticas relevantes que afectan significativamente sobre el bienestar psicológico y emocional de las personas que lo padecen. El objetivo de esta investigación fue analizar si las variables "regulación emocional" y "estados de estrés, depresión y ansiedad" están relacionadas con el ansia por la comida y la alimentación emocional. También se analizaron de forma complementaria las diferencias de sexo en las variables de estudio. El estudio se llevó a cabo con una muestra de 168 personas (46 hombres, 121 mujeres y 1 persona sin especificar), con un rango de edad de entre 18-73 años. Los resultados mostraron que la regulación emocional correlacionó negativamente con el ansia por la comida y la alimentación emocional. Igualmente se encontró correlación positiva con los estados de estrés y depresión. En cuanto al sexo, las mujeres presentaron una mayor alimentación emocional y estados de estrés y depresión, mientras que los hombres tuvieron relación significativa con la supresión expresiva. La discusión de estos resultados nos permite concluir que personas con una peor regulación emocional y expuestas a situaciones de estrés, depresión o ansiedad podrían tener más tendencia a experimentar ansia por la comida y alimentación emocional.

Palabra clave: Alimentación emocional

\section{Food craving and emotional eating: associated variables}

Abstract: Food craving and emotional eating are considered two relevant problems that significantly affect the psychological and emotional well-being of the people who suffer from it. The objective of this research was to analyze whether the variables "emotional regulation" and "states of stress, depression and anxiety" are related to the craving for food and emotional eating. Sex differences in the study variables were also analyzed in a complementary way. The study was carried out with a sample of 168 people (46 men, 121 women and 1 non-specified), with an age range between 18-73 years. The results showed that emotional regulation negatively correlated with food craving and emotional eating. Likewise, a positive correlation was found with states of stress and depression. Regarding sex, women presented greating emotional eating and states of stress and depression, while men had a significant relationship with expressive suppression. The discussion of these results allows us to conclude that people with poorer emotional regulation and exposed to situations of stress, depression or anxiety could be more likely to experience food craving and emotional eating.

Keyword: Emotional eating

\section{Introducción}

El ansia por la comida o deseo intenso de ingerir un determinado alimento, no puede clasificarse como un trastorno, ya que el impulso por comer un determinado alimento se da con mucha frecuencia en la población general. A pesar de que sea algo normal, hay veces que este deseo es tan irresistible e intenso que puede dar lugar a los atracones y a desarrollar comportamientos impulsivos hacia la comida (Water et al., 2001). El ansia por la comida se ha relacionado con el estado de ánimo, por lo que se ha planteado el término de "alimentación emocional", que se refiere al fenómeno de recurrir a la comida como forma de aliviar un sentimiento (Parker, 2006).

Investigaciones recientes, muestran que un $85 \%$ de las personas no poseen conocimientos suficientes acerca de las emociones o estados de ánimo y su impacto 
en la alimentación, lo cual sería importante para evitar enfermedades relacionadas con la mala alimentación tales como la obesidad, problemas cardíacos, colesterol, diabetes etc. (Peña \& Reidl, 2015).

La presente investigación se centra en el estudio del ansia por la comida y la alimentación emocional. Para ello, observaremos mediante diferentes cuestionarios algunas de las variables que se han asociado con esta problemática (e.g., regulación emocional, depresión y ansiedad) con el fin de examinar el grado de relación entre estas variables y los fenómenos de estudio en una muestra de población general, dado que, en su gran mayoría, esta asociación se ha fundamentado principalmente en muestras clínicas. (Montenegro, 2009)

\section{Ansia por la comida}

La investigación sobre el ansia es relativamente reciente y surgió asociada al ámbito de las adicciones (drug craving; Cepeda-Benito, 1993) Se considera que este deseo tiene una gran influencia en el mantenimiento de las conductas adictivas, siendo el responsable del uso compulsivo de la droga y el alto nivel de recaídas (Graña, 1994). Ya en 1948, Walker definió craving como la urgencia intensa y agobiante para el uso de opiáceos durante la abstinencia. Posteriormente, Tiffany (1995) lo describió como un estado motivacional (fisiológico y psicológico), que promueve la búsqueda de sustancias y la consiguiente realización de conductas de ingesta o consumo de esas sustancias, generalmente drogas o comida. A partir de ese momento, se ha manejado el término para definir problemas de adicciones a sustancias psicoactivas además del alcohol y tabaco (Cepeda et al., 2000). Los cravings, por lo tanto, tienen una gran influencia en el mantenimiento de las conductas adictivas, y por ello son responsables del uso compulsivo de la droga y del alto nivel de recaídas que sigue a cualquier tipo de tratamiento de las adicciones. A este respecto, se señala que es fundamental enseñar a los pacientes a analizar y afrontar cognitivamente sus deseos y observar cómo desaparecen sin necesidad de consumo (Sánchez-Hervás, 2001).

Paralelamente al concepto de craving, Hill y Brown en 1994 definieron por primera vez el food-craving como el impulso o deseo irrefrenable por consumir un determinado alimento. Más específicamente, el ansia por la comida se trata de un deseo intenso y prolongado de consumir un alimento determinado como un estado motivacional, con componentes fisiológicos y psicológicos, promoviendo la búsqueda de determinados alimentos para ingerirlos (Ables et al., 2011). Según Van der Ster Wallin (1994), este fenómeno ha sido relacionado con trastornos por atracones, con el abandono temprano de los tratamientos destinados a la pérdida de peso en personas obesas (Guertin, 1999) y con la larga duración de los TCAs (Gendall, 1997).

Una de las variables que pueden desencadenar el food-craving es el estado anímico negativo (Agras \& Telch, 1998). En este sentido, los resultados de un estudio realizado por Jáuregui-Lobera (2011) en una muestra de 65 personas estudiantes de la Universidad Pablo de Olavide de Sevilla mostraron que la ansiedad, depresión y estrategias de afrontamiento negativas influyeron en los resultados con respecto al food-craving inducido. De otro lado, existen investigaciones que apuntan a que un menor bienestar psicológico puede asociarse con el food-craving (Weingarten, 1990). En esta línea, un estudio reciente realizado por Ramón-Arbués (2019) en una muestra de 1055 estudiantes de diferentes titulaciones del campus de la Universidad San Jorge de Zaragoza, reveló cómo el ansia por la comida se relacionaba de forma significativa con la prevalencia de ansiedad, depresión y estrés. Es por ello que, el estrés, la 
ansiedad y depresión podrían dar lugar a ese deseo intenso por consumir un alimento determinado, en este caso no saludable.

\section{Alimentación emocional}

Al estudiar la conducta alimentaria, deben de tenerse en cuenta (entre otros) tres componentes individuales que afectan a la elección de los alimentos: cognitivo, conductual y afectivo (Herman \& Polivy, 1975). En relación con el componente afectivo (objeto de interés de la presente investigación), se ha observado que las emociones y estados de ánimo pueden influir en la conducta alimentaria, así como también la alimentación puede modificar los estados anímicos y emocionales (Gibson, 2006).

Clerget (2011) hace referencia al término "comedor emocional" como la persona que piensa en la comida cuando se sienten ansiosos, emotivos o negativos, tratando de impedir que se produzca una irrupción de sentimientos, pensamientos o emociones dolorosas. El comedor emocional, tiene dificultades para identificar sus emociones y la sensación real de hambre, así como una menor capacidad para expresar sus sentimientos. (Courbasson et al., 2008)

Diversos estudios ya identificaron que el comedor emocional incrementa el consumo de ciertos alimentos en respuesta a emociones desagradables (Macht., 2008); si bien el comedor no emocional no altera su consumo o incluso llega a restringirlo (Schachter et al., 1968). Concretamente, en el comedor emocional pueden encontrarse signos regidos por las emociones negativas como ingerir de forma impulsiva ciertos alimentos, consumirlos sin control (Batterink et al., 2010), continuar comiendo pese a sentirse lleno (Polivy, Coleman \& Herman, 2005), y sentimientos de culpa y remordimiento tras comer (Guerrieri et al., 2007). Más aún, estas personas parece que no pueden reconocer la razón por la que se alimentan así y más rápido de lo normal (Pelchat, 2002).

Además, el recurrir a la comida cuando uno está estresado, ansioso o por falta de tiempo hace que la persona prefiera alimentos con alto contenido calórico, ricos en azúcar, sal y grasa (Díaz Franco, 2007). A nivel físico, esto puede tener consecuencias como la diabetes, colesterol o problemas cardiovasculares (Dussaillant, 2015), mientras que a nivel psicológico puede haber perturbaciones que tienen que ver con la configuración mental de recurrir a este tipo de alimentación cada vez que experimenten emociones negativas (Rodríguez, 2009). Así, este impulso emocional se va a anteponer al hambre fisiológica o al hambre real, sin llegar a comprender que el bienestar solamente será temporal y no permanente (Whiteside et al, 2007).

\section{Influencia de la regulación emocional, depresión y ansiedad sobre la alimentación}

La regulación emocional es muy importante para mantener un autocontrol en la regulación del estado anímico, además de poder influir en la dieta y otras áreas de nuestra vida (Palomino-Pérez, 2020). Muchas veces, cuando queremos controlar una emoción negativa se recurre a la ingesta de alimentos, consumo de drogas, alcohol, etc. (Tice, 2000). Por lo tanto, el fin último de estas conductas es el de sentirse mejor, observándose en este sentido, una falta de autocontrol en su conducta alimentaria (Gross, 1999).

En relación con la regulación emocional, se han establecido dos dimensiones principales que han sido estudiadas y relacionadas con la alimentación (Montero \& León, 2007): la reevaluación cognitiva y la supresión expresiva (Goldin, 2009). En lo 
que refiere a la reevaluación cognitiva, se trata de una estrategia que modifica la forma de ver un evento cambiando su efecto emocional. Dicha estrategia permite disminuir experiencias emocionales negativas, demostrándose un factor de protección frente a la ansiedad y depresión (Andrés, 2013). Por su parte, la supresión expresiva es una reducción o inhibición de un estado emocional en particular. Se ha relacionado con disminución de la autoestima y de apoyo social (Gross, 1999), ya que solamente se produce una modificación fisiológica y de expresión de la emoción, la experiencia emocional no se ve disminuida (Oliver, 1999).

\section{Objetivos o hipótesis}

En base a lo expuesto en párrafos anteriores, la presente investigación tuvo como propósito examinar la relación entre el ansia por la comida, la alimentación emocional y algunas variables que pueden estar asociadas con estos fenómenos, como pueden ser la regulación emocional o el estrés, depresión y ansiedad. En cuanto a las hipótesis de este estudio, se espera encontrar, por un lado, una asociación negativa entre la regulación emocional y el ansia por la comida (Hipótesis 1a), y una relación positiva entre el ansia por la comida y estados de depresión y ansiedad (Hipótesis 1b). Asimismo, se espera encontrar una relación negativa entre la regulación emocional y alimentación emocional (Hipótesis 2a), y una asociación positiva entre la alimentación emocional y estados de depresión y ansiedad (Hipótesis 2b).

Como un objetivo adicional de esta investigación, se examinaron las diferencias de sexo en cada una de las variables de estudio con el fin de observar si nuestros datos apuntan en la línea de investigaciones previas, las cuales sugieren que son las mujeres (vs. hombres) quienes presentan en mayor grado una pobre regulación emocional (Lane 2000), una mayor ansia por la comida (Polivy, 2005), alimentación emocional (Ramos López, 2018), depresión y ansiedad (Lazarevich, 2018).

\section{Método}

\section{Participantes}

La muestra inicial estuvo formada por un total de 247 personas, de las cuales 79 se eliminaron porque no completaron la totalidad del cuestionario (52 hombres y 27 mujeres; edad comprendida entre 18-73 años; $M=23,70 ; D T=9,42$ ). Por tanto, la muestra final se conformó de 168 personas (46 hombres, 121 mujeres y 1 persona sin especificar), con un rango de edad que oscila entre los 18-73 años $(M=24,90 ; D T=$ 10,63 ). Además de su edad y el género, los participantes han informado de otras variables, en este caso su estado civil y si han tenido alguna experiencia estresante 0 traumática a lo largo de su vida. El $58.9 \%$ de los participantes se encontraban solteros/as, un $31 \%$ en relación de pareja, un $7.7 \%$ casados/as, el $1.8 \%$ divorciado/a y el $0.6 \%$ viudo/a. Por otro lado, el $59.5 \%$ de los participantes han experimentado a lo largo de su vida alguna experiencia estresante o traumática mientras el restante $40.5 \%$ no han sufrido una experiencia similar.

\section{Instrumentos}

Cuestionario de Regulación Emocional (ERQ; Gross y John, 1998; adaptación en su versión al castellano por Rodríguez-Carvajal et al., 2006). Este instrumento mide las variables de control o manejo y regulación de emociones mediante las subescalas de reevaluación cognitiva (e.g., "Cuando quiero incrementar mis emociones positivas (p.ej. alegría, diversión), cambio el tema sobre el que estoy pensando") y supresión expresiva (e.g., "Guardo mis emociones para mí mismo/a"). Este cuestionario consta 
de 10 preguntas puntuables del 1 al $7(1=$ totalmente en desacuerdo; $7=$ totalmente de acuerdo).

La consistencia interna del cuestionario en el estudio fue de 0.650 ( $\alpha$ de Cronbach)

Cuestionario breve de Depresión y Ansiedad (PHQ-4; Kroenke et al., 2009 adaptación en su versión al castellano por Diez-Quevedo et al., 2001). Este instrumento consta de un total de 4 ítems: 2 ítems que evalúan la depresión (e.g., "Me siento de bajón, deprimido/a o desesperado/a") y 2 ítems que evalúan la ansiedad (e.g., "Me siento nervioso/a, ansioso/a o tenso/a"). Tiene cuatro categorías de respuesta: 0 (nunca), 1 (varios días), 2 (más de la mitad de los días) y 3 (casi todos los días), demostrando tener buenas propiedades psicométricas para detectar los trastornos del estado de ánimo y de ansiedad.

La consistencia interna del cuestionario en el estudio fue de 0.812 ( $\alpha$ de Cronbach)

Cuestionario de Comedor Emocional Garaulet (CCE; Garaulet, et al., 2012). Esta medida clasifica a las personas en función de la relación entre la ingesta de alimento y las emociones. Está conformado por 10 ítems (e.g., "¿Tienes antojos por ciertos alimentos específicos?") con un formato de respuesta tipo Likert de cuatro alternativas $(0=$ nunca, $1=$ a veces, $2=$ generalmente y $3=$ siempre $)$, de modo que, a mayor puntuación, mayor es también el efecto de las emociones sobre la elección de alimentos y los hábitos alimentarios.

La consistencia interna del cuestionario en el estudio fue de 0.865 ( $\alpha$ de Cronbach)

Cuestionario de Ansia por la Comida como Rasgo (Food Craving QuestionnaireTrait FCQ-T; validación en su versión al castellano por Moreno et al., 2009). Este instrumento mide el deseo de comer característico de cada participante (cravings de rasgo). El cuestionario consta de 39 ítems (e.g., "A veces, cuando como lo que se me antoja, pierdo el control y como demasiado"), empleando una escala de 6 puntos donde el sujeto debe indicar con un número del 1 al 6 si las situaciones planteadas le suceden ( $1=$ Nunca, $2=$ Raramente, $3=$ Algunas veces, $4=A$ menudo, $5=$ Casi siempre y $6=$ Siempre).

La consistencia interna del cuestionario en el estudio fue de 0.961 ( $\alpha$ de Cronbach)

\section{Procedimiento}

Antes de llevar a cabo la investigación, se informó a los participantes del carácter voluntario del estudio y el anonimato y confidencialidad de sus respuestas, sin recibir compensación económica por su participación. A través de la plataforma LimeSurvey, se ha evaluado el manejo de emociones mediante el cuestionario, la depresión y ansiedad, la relación entre la ingesta de alimentos y emociones, y el ansia por la comida.

A cada participante se les mostró en la plataforma web una hoja de información y unas instrucciones básicas respecto a cómo completar cada cuestionario. De igual forma se pidió el consentimiento informado.

La investigación se llevó a cabo tras recibir la aprobación del Comité de Ética de la Universidad de Granada.

\section{Análisis estadísticos}


Todos los datos del estudio se han analizado con el paquete SPSS (IBM SPSS Statistics 27.0). Para analizar la relación que existe entre el ansia por la comida, la alimentación emocional, y la regulación emocional y estados de depresión y ansiedad se empleó un análisis de correlaciones bivariadas. Asimismo, para comprobar si existían diferencias de sexo en cada una de las variables de estudio, se llevó a cabo una prueba t-student para muestras independientes.

\section{Resultados}

\section{Asociaciones entre el ansia por la comida, alimentación emocional, estados de depresión $y$ ansiedad $y$ regulación emocional (reevaluación cognitiva $y$ supresión expresiva).}

Los resultados muestran que hay una correlación positiva entre el ansia por la comida y la supresión expresiva, por lo que, a peor regulación emocional, mayor ansia por la comida (Hipótesis 1a). De igual modo, se obtuvo una asociación positiva entre el ansia por la comida y estados de estrés y ansiedad. (Hipótesis 1b), lo que resulta indicativo de que, a mayor estrés y ansiedad, mayor ansia por la comida.

Asimismo, la alimentación emocional se ha asociado positivamente con la supresión expresiva, por lo que una peor regulación emocional se asocia a un mayor nivel de alimentación emocional (Hipótesis 2a).

Por último, para la alimentación emocional también se encontró correlación positiva con estados de estrés y depresión (Hipótesis $2 \mathrm{~b}$ ), indicando que mayores niveles de estrés y depresión están relacionados con altos niveles de alimentación emocional.

Los resultados confirman las hipótesis 1 y 2 . (ver tabla 1 ).

Tabla 1. Estadística descriptiva y correlaciones entre las variables principales del estudio

\begin{tabular}{|c|c|c|c|c|c|c|c|c|}
\hline & 1 & 2 & 3 & 4 & 5 & 6 & 7 & 8 \\
\hline 1. Ansia por la comida & -------- & & & & & & & \\
\hline 2.Alimentación emocional & $0.798^{* *}$ & ------- & & & & & & \\
\hline 3. Supresión expresiva & $0.210^{\star *}$ & $0.157^{*}$ & ------- & & & & & \\
\hline 4.Reevaluación cognitiva & 0.006 & -0.003 & $0.491^{* *}$ & ------ & & & & \\
\hline 5. Estrés, Depresión y Ansiedad & $0.403^{\star \star}$ & $0.358^{* *}$ & $0.208^{* *}$ & -0.083 & ------- & & & \\
\hline 6. Sexo ${ }^{a}$ & $0.161^{*}$ & $0.188^{*}$ & $-0.268^{\star *}$ & -0.037 & $0.169^{*}$ & ------ & & \\
\hline 7. Edad & -0.074 & 0.038 & 0.022 & -0.121 & -0.062 & ------ & ------- & \\
\hline 8. Experiencia traumática ${ }^{b}$ & -0.019 & -0.089 & -0.085 & -0.008 & $-0.205^{\star *}$ & 0.032 & $-0.191^{*}$ & ------ \\
\hline M & 106.39 & 9.75 & 3.91 & 4.57 & 4.82 & ------- & 24.90 & ------ \\
\hline DE & 33.41 & 6.04 & 0.92 & 0.87 & 2.80 & ------ & 10.65 & ----- \\
\hline
\end{tabular}

Nota. $M=$ Media, $D E=$ Desviación Estándar; a $0=$ hombre, $1=$ mujer; $b 0=$ sí, $1=n o ;{ }^{*} p<0.05,{ }^{* *} p<0.01$

\section{Análisis auxiliares}

Para observar el papel del sexo en las diferentes variables de este estudio, se llevó a cabo una prueba T-Student de muestras independientes. El sexo de los participantes fue incluido como variable independiente, mientras que el ansia por la comida, alimentación emocional, estados de estrés y depresión y regulación emocional (reevaluación cognitiva y supresión expresiva) se utilizaron como variables dependientes. Se codificó el sexo como 0 para hombre y 1 para mujeres. Los resultados mostraron diferencias estadísticamente significativas en alimentación emocional y estados de estrés y depresión según el sexo, siendo las mujeres quienes tuvieron una puntuación mayor en alimentación emocional y el estado de estrés y 
depresión. Por otro lado, también se obtuvo diferencias estadísticamente significativas para la supresión expresiva, siendo los hombres quienes obtuvieron una mayor puntuación (ver tabla 2).

Tabla 2. Diferencias de sexo en las principales variables del estudio

\begin{tabular}{|c|c|c|c|c|c|c|}
\hline & $\begin{array}{l}\text { Hombres } \\
M(D E)\end{array}$ & $\begin{array}{l}\text { Mujeres } \\
\text { M (DE) }\end{array}$ & $t$ & $p$ & $95 \%$ IC & $\mathrm{d}$ \\
\hline 1. Ansia por la comida & $97.82(35.54)$ & $109.50(32.24)$ & -1.945 & 0.056 & {$[-23.64,0.28]$} & -0.352 \\
\hline 2.Alimentación emocional & $7.84(5.86)$ & $10.47(6.00)$ & -2.575 & 0.012 & {$[-4.66,-0.59]$} & -0.423 \\
\hline 3.Reevaluación cognitiva & $4.63(1.10)$ & $4.54(0.77)$ & 0.505 & 0.556 & {$[-0.26,0.44]$} & 0.102 \\
\hline 4. Supresión expresiva & $4.31(0.99)$ & $3.76(0.85)$ & 3.354 & 0.001 & {$[0.22,0.88]$} & 0.620 \\
\hline $\begin{array}{r}\text { 5. Estrés, Depresión y } \\
\text { Ansiedad }\end{array}$ & $3.95(2.23)$ & $5.19(2.91)$ & -2.920 & 0.004 & {$[-2.07,-0.39]$} & -0.449 \\
\hline
\end{tabular}

Nota. $M=$ Media, $D E=$ Desviación Estándar; $d=d$ de Cohen

\section{Discusión}

Esta investigación trató de examinar la relación entre variables como regulación emocional, estados de estrés y depresión, y el ansia por la comida y alimentación emocional.

Los resultados revelaron que una baja regulación emocional y un mayor estrés y depresión se relacionan con mayor ansia por la comida. La regulación emocional, es clave para mantener el autocontrol en muchos ámbitos de nuestra vida y cambios en el estado de ánimo y/o sentimientos, pueden influir en la dieta, control de impulsos etc. (Gross, 1999). En relación con los estados de depresión y ansiedad se ha demostrado que los sucesos estresantes de la vida cotidiana pueden provocar una sensibilización crónica de las áreas subcorticales que se encuentran a la base del impulso por la comida (Lyvers, 2000).

Respecto a la alimentación emocional, los resultados mostraron que una peor regulación de las emociones se asocia a una mayor alimentación emocional. Además, se encontró también asociación positiva entre los estados de estrés y depresión y la alimentación emocional, por lo que, a mayor estrés y depresión, mayor alimentación emocional. Se considera que el comer en exceso se encuentra vinculado a las emociones negativas. En este caso, la alimentación emocional nace como respuesta a los estados de ánimo negativos con el fin de aliviar el estrés y estas emociones (Bekker et al., 2003).

Como objetivo adicional, los resultados de los análisis auxiliares revelaron que las mujeres presentaron mayores niveles de alimentación emocional, y de estrés y depresión. Esto puede ser debido al exceso de cortisol, un factor que suele predominar en mujeres y que puede afectar de forma directa al hambre emocional (Cortés Romero, 2008). El aumento excesivo del estrés a nivel fisiológico y psicológico desencadenará una serie de alteraciones fisiológicas que dan lugar a una liberación de cortisol plasmático (Dress et al, 2017). Hay estudios que dudan si el manejo de las dos estrategias de regulación emocional (reevaluación cognitiva y supresión expresiva) es un tema de identidad de género y no biológico (Wester, 2002). 
El presente estudio muestra la importancia de nuestro estado de ánimo y emociones en la alimentación además de la relación de estas variables con el estrés, depresión y el ansia por la comida. Sería interesante integrar en programas destinados a controlar la alimentación emocional y el ansia por la comida estrategias de regulación emocional y para trabajar el estrés, depresión y ansiedad.

Este estudio presenta también varias limitaciones. En primer lugar, la muestra del estudio podría ser mayor para poder obtener resultados aún más significativos, por lo que limita la capacidad de generalizar a una población más amplia. Otra limitación de este estudio es la desproporción entre hombres y mujeres en la muestra, ya que como análisis auxiliares se ha realizado una valoración de las diferencias de sexo en las principales variables del estudio.

Futuros estudios podrían atender a estas limitaciones a través de una muestra mayor y una mejor proporción de género.

\section{Conclusión}

Este estudio contribuye a una mejor comprensión del área de investigación del ansia por la comida y la alimentación emocional, revelando que una mala regulación emocional (mayor supresión expresiva) y el estrés, depresión y ansiedad pueden dar lugar a una mayor ansia por la comida y alimentación emocional.

Aunque los resultados de este estudio sean preliminares, estos hallazgos podrían ser importantes para la elaboración de estrategias que permitan controlar y trabajar de alguna forma la relación entre las variables descritas en esta investigación, el ansia por la comida y la alimentación emocional. Por último, también puede resultar positivo, la realización de nuevos estudios que relacionen estas variables con el género, ya que en este estudio no se ha podido conseguir una muestra proporcionada y ha sido difícil contar con bibliografía que vaya en esta línea.

\section{Referencias}

Ables, E. T., y Drummond-Barbosa, D. (2011). Food for thought: neural stem cells on a diet. Cell Stem Cell, 8(4), 352-354.

Agras, W. S., y Telch, C. F. (1998). The effects of caloric deprivation and negative affect on binge eating in obese bingeeating disordered women. Behavior Therapy, 29(3), 491-503.

Andrés, M. L., y Aydmune, Y. (2013). Control cognitivo de la emoción: funciones ejecutivas implicadas en el uso de la reevaluación cognitiva como estrategia de regulación emocional.

Batterink, L., Yokum, S., y Stice, E. (2010). Body mass correlates inversely with inhibitory control in response to food among adolescent girls: an fMRI study. Neuroimage, 52(4), 1696-1703.

Bekker, M. H., Van De Meerendonk, C., y Mollerus, J. (2004). Effects of negative mood induction and impulsivity on selfperceived emotional eating. International Journal of Eating Disorders, 36(4), 461-469.

Cepeda-Benito, A. (1993). Meta-analytical review of the efficacy of nicotine chewing gum in smoking treatment programs. Journal of consulting and clinical psychology, 61(5), 822.
Cepeda-Benito, A., Gleaves, D. H., Williams, T. L., y Erath, S. A. (2000). The development and validation of the state and trait foodcravings questionnaires. Behavior therapy, 31(1), 151-173.

Clerget, S. (2011). Sobrepeso Emocional. México: Urano.

Courbasson, C. M., Rizea, C., y Weiskopf, N. (2008). Emotional eating among individuals with concurrent eating and substance use disorders. International Journal of Mental Health and Addiction, 6(3), 378-388.

Díaz Franco, J. J. (2007). Estrés alimentario y salud laboral vs. estrés laboral y alimentación equilibrada. Medicina y seguridad del trabajo, 53(209), 93-99.

Dussaillant, C., Echeverría, G., Villarroel, L., Marin, P. P., y Rigotti, A. (2015). Una alimentación poco saludable se asocia a mayor prevalencia de síndrome metabólico en la población adulta chilena: estudio de corte transversal en la Encuesta Nacional de Salud 2009-2010. Nutrición Hospitalaria, 32(5), 2098-2104.

Gendall, K. A., Joyce, P. R., y Sullivan, P. F. (1997). Impact of definition on prevalence of food cravings in a random sample of young women. Appetite, 28(1), 63-72. 
Gibson, E. (2006). Emotional influences on food choice: Sensory, physiological and psychological pathways. Physiology \& Behavior, $89,53-61$.

Goldin, P. R., Manber-Ball, T., Werner, K., Heimberg, R., y Gross, J. J. (2009). Neural mechanisms of cognitive reappraisal of negative self-beliefs in social anxiety disorder. Biological psychiatry, 66(12), 1091-1099.

Graña Gómez, J. L., y García Álvarez, Á. (1994). Prevención de recaídas en drogodependencias. Graña Gómez, JL (1994). Conductas adictivas: teoría, evaluación y tratamiento. Madrid: Debate, 225-259.

Gross, J. J. (1999). Emotion and emotion regulation. Handbook of personality: Theory and research, 2, 525-552.

Guerrieri, R., Nederkoorn, C., Stankiewicz, K., Alberts, H., Geschwind, N., Martijn, C., y Jansen, A. (2007). The influence of trait and induced state impulsivity on food intake in normal-weight healthy women. Appetite, 49(1), 66-73.

Guertin, T. L. (1999). Eating behavior of bulimics, self-identified binge eaters, and non-eating-disordered individuals: what differentiates these populations?. Clinical psychology review, 19(1), $1-23$.

Herman, C. P., y Polivy, J. (1975). Anxiety, restraint, and eating behavior. Journal of abnormal psychology, 84(6), 666.

Hill, A. J., y Heaton-Brown, L. (1994). The experience of food craving: A prospective investigation in healthy women. Journal of Psychosomatic Research, 38(8), 801-814.

Lane, A. M., y Terry, P. C. (2000). The nature of mood: Development of a conceptual model with a focus on depression. Journal of applied sport psychology, 12(1), 16-33.

Lazarevich, I., Camacho, M. E. I., Alva, M. D. C. V., Flores, N. L., Medina, O. N., y Zepeda, M. A. Z. (2018). Depression and food consumption in Mexican college students. Nutrición hospitalaria: Organo oficial de la Sociedad española de nutrición parenteral y enteral, 35(3), 620-626.

Lobera, I. J., Cid, S. T., y Ríos, P. B. (2011). Body shape model, physical activity and eating behaviour. Nutricion hospitalaria, 26(1), 201-207.

López, R. S., Maciá, C. G., y Juan, M. V. (2018). Inteligencia emocional en alumnado de formación profesional. Diferencias en función del curso, del género y de la edad. Educar, 54(1), 229-245.

Lyvers, M. (2000). " Loss of control" in alcoholism and drug addiction: A neuroscientific interpretation. Experimental and clinical psychopharmacology, 8(2), 225.

Macht, M. (2008). How emotions affect eating: a five-way model. Appetite, 50(1), 1-11.

Montero, I., y León, O. G. (2007). A guide for naming research studies in Psychology. International Journal of clinical and Health psychology, 7(3), 847-862.

Oliver, G., y Wardle, J. (1999). Perceived effects of stress on food choice. Physiology \& behavior, 66(3), 511-515.

Palomino-Pérez, A. M. (2020). Rol de la emoción en la conducta alimentaria. Revista chilena de nutrición, 47(2), 286-291.
Parker, G., Parker, I., Brotchie, H., y Stuart, S. (2006). Interpersonal psychotherapy for depression? The need to define its ecological niche. Journal of Affective Disorders, 95(1-3), 1-11.

Pelchat, M. L. (2002). Of human bondage: food craving, obsession, compulsion, and addiction. Physiology \& behavior, 76(3), 347-352.

Peña Fernández, E., y Reidl Martínez, L. M. (2015). Las emociones y la conducta alimentaria. Acta de investigación psicológica, 5(3), 2182-2193.

Polivy, J., Coleman, J., y Herman, C. P. (2005). The effect of deprivation on food cravings and eating behavior in restrained and unrestrained eaters. International Journal of Eating Disorders, 38(4), 301-309.

Ramón Arbués, E., Martínez Abadía, B., Granada López, J. M., Echániz Serrano, E., Pellicer García, B., Juárez Vela, R., y Sáez Guinoa, M. (2019). Conducta alimentaria y su relación con el estrés, la ansiedad, la depresión y el insomnio en estudiantes universitarios. Nutrición Hospitalaria, 36(6), 1339-1345.

Rodríguez, J. A. P., Linares, V. R., González, A. E. M., y Guadalupe, L. A. O. (2009). Emociones negativas y su impacto en la salud mental y física. Suma psicológica, 16(2), 85-112.

Romero, C. E. C., Noriega, A. E., Ruiz, J. C., Rodríguez, G. S., Reboredo, T. B., y Pliego, M. V. (2018). Stress and cortisol: implications on food intake. Revista Cubana de Investigaciones Biomédicas, 37(3), 1-15.

Sánchez-Hervás, E., Gradolí, V. T., y Gallús, E. M. (2004). Un modelo de tratamiento psicoterapéutico en adicciones. Trastornos adictivos, 6(3), 159-166.

Schachter, S., Goldman, R., y Gordon, A. (1968). Effects of fear, food deprivation, and obesity on eating. Journal of personality and social psychology, 10(2), 91.

Tice, D. M., \& Bratslavsky, E. (2000). Giving in to feel good: The place of emotion regulation in the context of general self-control. Psychological inquiry, 11(3), 149-159.

Tiffany, S. T., Carter, B. L., y Singleton, E. G. (2000). Challenges in the manipulation, assessment and interpretation of craving relevant variables. Addiction, 95(8s2), 177-187.

Van der Ster Wallin, G., Norring, C., y Holmgren, S. (1994). Binge eating versus non-purged eating in bulimics: is there a carbohydrate craving after all?. Acta Psychiatrica Scandinavica, 89(6), 376-381.

Walker, S. J., Goldschmidt, D., y Ribeiro, C. (2017). Craving for the future: the brain as a nutritional prediction system. Current opinion in insect science, 23, 96-103

Waters, A., Hill, A., y Waller, G. (2001). Bulimics' responses to food cravings: is binge-eating a product of hunger or emotional state?. Behaviour Research and Therapy, 39(8), 877-886.

Weingarten, H. P., y Elston, D. (1990). The phenomenology of food cravings. Appetite, 15(3), 231-246.

Wester, S. R., Vogel, D. L., Pressly, P. K., y Heesacker, M. (2002). Sex differences in emotion: A critical review of the literature and implications for counseling psychology. The Counseling Psychologist, 30(4), 630-652. 
Whiteside, U., Chen, E., Neighbors, C., Hunter, D., Lo, T., y Larimer, M. (2007). Difficulties regulating emotions: Do binge eaters have fewer strategies to modulate and tolerate negative affect? Eating Behavior, 2, 162-169. 